International Mathematical Forum, 1, 2006, no. 29, 1441 - 1451

\title{
On common fixed point theorems in fuzzy metric spaces
}

\author{
Seong Hoon Cho \\ Department of Mathematics, \\ Hanseo University, \\ Seosan, Chungnam, 356-820, South Korea \\ Ji Hong Jung \\ Department of Mathematics, \\ Hanseo University, \\ Seosan, Chungnam, 356-820, South Korea
}

\begin{abstract}
In this paper we introduce the notion of $\epsilon$-chanable fuzzy metirc space. We give some conditions of which four self mappings of $\epsilon$-chanable fuzzy metric space have a unique common fixed point. Also we characterize the conditions for two self mappings of $\epsilon$-chanable fuzzy metric space have a unique common fixed point.
\end{abstract}

Mathematics Subject Classification: 47H10, 54H25

Keywords: fuzzy metric space, $\epsilon$-chanable fuzzy metric space, compatible mappings, weakly compatible mappings, common fixed point

\section{Introduction}

The concept of fuzzy sets was introduced initially by Zadeh[21]. Since then, it was developed extensively by many authors and used in various fields. Especially, $[2,6,12,14]$ introduced the concept of fuzzy metric spaces in different ways. In $[6,7]$, George and Veeramani modified the concept of fuzzy metric space which introduced by Kramosil and Michalek[14]. They, also, obtained the Hausdorff topology for this kind of fuzzy metric spaces and showed that every metric induces a fuzzy metric.

Sessa[17] introduced a generalization of commutativity, so called weak commutativity. Further Jungck[10] introduced more generalized commutativity, 
which is called compatibility in metric space and proved common fixed point theorems. Grabiec[8] proved fuzzy Banach contraction theorem on fuzzy metric space in the sence of [14]. Bijendra Singh and M. S. Chauhan[18] introduced the concept of compatibility in fuzzy metric space and proved some common fixed point theorems in fuzzy metric spaces in the sence of George and Veeramani. Jungck and Rhoades[11] introduced the notion of coincidentally commuting(or weakly compatible) mappings and obtained fixed point theorems for set-valued mapping. Also, Dhage[3] introduced this concept and proved common fixed point theorems in D-metric space.

Recently, Bijendra Singh and Shishir Jain[19] introduced the concept of weak compatibility in Menger space and proved common fixed point theorem in Menger space.

In this paper, we introduce the concept of $\epsilon$-chainable fuzzy metric space and prove common fixed point theorems for four weakly compatible mappings of $\epsilon$-chainable fuzzy metric space. We, also, characterize the conditions for self mappings of $\epsilon$-chainable fuzzy metric space have a unique common fixed point which is a extension of the result of [5] and we extend a result of [15] to $\epsilon$-chainable fuzzy metric space.

\section{Prelimimaries}

In this section, we give some definitions and lemmas.

Definition 2.1. [16] A binary operation $*:[0,1] \times[0,1] \rightarrow[0,1]$ is called a continuous $t$-norm if $([0,1], *)$ is an abelian topological monoid with 1 such that $a * b \leq c * d$, whenever $a \leq c, b \leq d$ for all $a, b, c, d \in[0,1]$.

Examples of $t$-norm are $a * b=a b$ and $a * b=\min \{a, b\}$.

Definition 2.2. [6] The 3-tuple $(X, M, *)$ is called a fuzzy metric space if $X$ is an arbitrary set, $*$ is a continuous $t$-norm and $M$ is a fuzzy set on $X^{2} \times(0, \infty)$ satisfying the following conditions:

(1) $M(x, y, t)>0$,

(2) $M(x, y, t)=1$ if and only if $x=y$,

(3) $M(x, y, t)=M(y, x, t)$,

(4) $M(x, y, t) * M(y, z, s) \leq M(x, z, t+s)$,

(5) $M(x, y, \cdot):(0, \infty) \rightarrow[0,1]$ is continuous,

for all $x, y, z \in X$ and $t, s>0$.

Let $(X, d)$ be a metric space, and let $a * b=a b$ or $a * b=\min \{a, b\}$. Let $M(x, y, t)=\frac{t}{t+d(x, y)}$ for all $x, y \in X$ and $t>0$. Then $(X, M, *)$ is a fuzzy metric space, and this fuzzy metric $M$ induced by $d$ is called the standard fuzzy metric[6]. 
Definition 2.3. [8] A sequence $\left\{x_{n}\right\}$ in a fuzzy metric space $(X, M, *)$ is said to be convergent to a point $x \in X$ (denoted by $\left.\lim _{n \rightarrow \infty} x_{n}=x\right)$, if for each $\epsilon>0$ and each $t>0$, there exists $n_{0} \in \mathbb{N}$ such that $M\left(x_{n}, x, t\right)>1-\epsilon$ for all $n \geq n_{0}$.

George and Veeramani[6] show that a sequence $\left\{x_{n}\right\}$ in a fuzzy metric space $(X, M, *)$ converges to a point $x \in X$ if and only if $\lim _{n \rightarrow \infty} M\left(x_{n}, x, t\right)=1$ for all $t>0$.

A sequence $\left\{x_{n}\right\}$ in a fuzzy metric space $(X, M, *)$ is called Cauchy sequence[8] if for each $\epsilon>0$ and each $t>0$, there exists $n_{0} \in \mathbb{N}$ such that $M\left(x_{n}, x_{n+p}, t\right)>1-\epsilon$ for all $n \geq n_{0}$ and all $t>0$.

George and Veeramani[6] gave an example that $(\mathbb{R}, M, *)$ is not complete in the sense of [8], where $M$ is the standard fuzzy metric with $d(x, y)=|x-y|$, and so to make $\mathbb{R}$ complete fuzzy metric space George and Veeramani redefine Cauchy sequence as follows.

Definition 2.4. [6] A sequence $\left\{x_{n}\right\}$ in a fuzzy metric space $(X, M, *)$ is called Cauchy if for each $\epsilon>0$ and $t>0$, there exists $n_{0} \in \mathbb{N}$ such that $M\left(x_{n}, x_{m}, t\right)>1-\epsilon$ for all $n, m \geq n_{0}$.

A fuzzy metric space in which every Cauchy sequene is convergent is said to be complete.

Guangxing Song[20] gave an example that the definition of Cauchy sequence in the sence of [8] is incorrect and modified the definition of Cauchy sequence in the sence of [8] as follows.

A sequence $\left\{x_{n}\right\}$ in a fuzzy metric space $(X, M, *)$ is Cauchy sequence if and only if $M\left(x_{n}, x_{n+p}, t\right) \rightarrow 1$ (for each $t>0$ ) as $n \rightarrow \infty$ unifomly on $p \in \mathbb{N}$.

Guangxing Song[20] proved that the meanings of definitions of Cauchy sequence in the sence of [20] and [6] are same.

Definition 2.5. [18] Self mappings $A$ and $B$ of a fuzzy metric space $(X, M, *)$ is said to be compatible if $\lim _{n \rightarrow \infty} M\left(A B x_{n}, B A x_{n}, t\right)=1$ for all $t>0$, whenever $\left\{x_{n}\right\}$ is a sequence in $X$ such that $\lim _{n \rightarrow \infty} A x_{n}=\lim _{n \rightarrow \infty} B x_{n}=z$ for some $z \in X$.

Definition 2.6. [1] Self mappings $A$ and $B$ of a fuzzy metric space $(X, M, *)$ is said to be weakly compatible if $A B x=B A x$ when $A x=B x$ for some $x \in X$.

It is easy to see that if self mappings $A$ and $B$ of a fuzzy metric space $(X, M, *)$ is compatible then they are weakly compatible.

The following example shows that the converse of above statement does not hold.

Example Let $X=[0,2]$ and $a * b=\min \{a, b\}$. Let $M$ be the standard fuzzy metric induced by $d$, where $d(x, y)=|x-y|$ for $x, y \in X$. 
Define two self mappings $A$ and $B$ of fuzzy metric space $(X, M, *)$ as follows:

$$
A x=\left\{\begin{array}{ll}
2-x, & (0 \leq x \leq 1) \\
2, & (1<x \leq 2)
\end{array} \text { and } \quad B x= \begin{cases}x, & (0 \leq x \leq 1) \\
2, & (1<x \leq 2)\end{cases}\right.
$$

Let $x_{n}=1-\frac{1}{n}$. Then $[A, B]$ is not compatible but $[A, B]$ is weakly compatible(see [1]).

Let $(X, M, *)$ be a fuzzy metric space and $\epsilon>0$. A finite sequence $x=$ $x_{0}, x_{1}, \cdots, x_{n}=y$ is called $\epsilon$-chain from $x$ to $y$ if $M\left(x_{i}, x_{i-1}, t\right)>1-\epsilon$ for all $t>0$ and $i=1,2, \cdots, n$.

A fuzzy metric space $(X, M, *)$ is called $\epsilon$-chainable if for any $x, y \in X$, there exists an $\epsilon$-chain from $x$ to $y$.

Lemma 2.7. [8] Let $(X, M, *)$ be a fuzzy metric space. Then for all $x, y \in$ $X, M(x, y, \cdot)$ is nondecreasing

From now on, let $(X, M, *)$ be a fuzzy metric space such that $\lim _{t \rightarrow \infty} M(x, y, t)=1$ for all $x, y \in X$ and $t * t \geq t$ for all $t \in[0,1]$.

Let $T$ and $T_{M}$ be two $t$-norm defined by for all $t, a, b \in[0,1], T(t, t)=t * t \geq$ $t$ and $T_{M}(a, b)=\min \{a, b\}$, respectively. Then we know that the minimum $T_{M}$ is the strongest $t$-norm(see [13]).

Guangxing Song considered as $T \neq T_{M}$ in [20], but it is well known that[13] $T=T_{M}$, that is, the only $t$-norm satisfying $T(t, t)=t * t \geq t$ for all $t \in[0,1]$ is the minimum $T_{M}$.

Lemma 2.8. Let $(X, M, *)$ be a fuzzy metric space. If there exists $q \in(0,1)$ such that $M(x, y, q t) \geq M(x, y, t)$ for all $x, y \in X$ and $t>0$, then $x=y$.

Proof. Suppose that there exists $q \in(0,1)$ such that $M(x, y, q t) \geq M(x, y, t)$ for all $x, y \in X$ and $t>0$. Then $M(x, y, t) \geq M\left(x, y, \frac{t}{q}\right)$ and so $M(x, y, t) \geq$ $M\left(x, y, \frac{t}{q^{n}}\right)$ for positive integer $n$. Taking limit as $n \rightarrow \infty, M(x, y, t) \geq 1$ and hence $x=y$.

\section{Main Results}

Theorem 3.1. Let $(X, M, *)$ be a complete $\epsilon$-chainable fuzzy metric space and let $A, B, S$ and $T$ be self mappings of $X$ satisfying the following conditions:

(i) $A X \subset T X$ and $B X \subset S X$,

(ii) $A$ and $S$ are continuous,

(iii) the pairs $[A, S]$ and $[B, T]$ are weakly compatible, 
(iv) there exists $q \in(0,1)$ such that

$$
M(A x, B y, q t) \geq M(S x, T y, t) * M(A x, S x, t) * M(B y, T y, t) * M(A x, T y, t)
$$

for every $x, y \in X$ and $t>0$.

Then $A, B, S$ and $T$ have a unique common fixed point in $X$.

Proof. As in [17], we can find a Cauchy sequence $\left\{y_{n}\right\}$ in $X$ such that

$y_{2 n-1}=T x_{2 n-1}=A x_{2 n-2}$ and $y_{2 n}=S x_{2 n}=B x_{2 n-1}$ for $n=1,2 \cdots$.

From completeness, $y_{n} \rightarrow z$ for some $z \in X$, and so $\left\{A x_{2 n-2}\right\},\left\{S x_{2 n}\right\}$, $\left\{B x_{2 n-1}\right\}$ and $\left\{T x_{2 n-1}\right\}$ also converges to $z$.

Since $X$ is $\epsilon$-chainable, there exists $\epsilon$-chain from $x_{n}$ to $x_{n+1}$, that is, there exists a finite sequence $x_{n}=y_{1}, y_{2}, \cdots, y_{l}=x_{n+1}$ such that $M\left(y_{i}, y_{i-1}, t\right)>1-$ $\epsilon$ for all $t>0$ and $i=1,2, \cdots, l$. Thus we have $M\left(x_{n}, x_{n+1}, t\right) \geq M\left(y_{1}, y_{2}, t / l\right) *$ $M\left(y_{2}, y_{3}, t / l\right) * \cdots * M\left(y_{l-1}, y_{l}, t / l\right)>(1-\epsilon) *(1-\epsilon) * \cdots *(1-\epsilon) \geq 1-\epsilon$.

For $m>n, M\left(x_{n}, x_{m}, t\right) \geq M\left(x_{n}, x_{n+1}, t / m-n\right) * M\left(x_{n+1}, x_{n+2}, t / m-n\right) *$ $\cdots * M\left(x_{m-1}, x_{m}, t / m-n\right)>(1-\epsilon) *(1-\epsilon) * \cdots *(1-\epsilon)>1-\epsilon$, and so $\left\{x_{n}\right\}$ is a Cauchy sequence in $X$ and hence there exists $x \in X$ such that $x_{n} \rightarrow x$. From $(i i), A x_{2 n-2} \rightarrow A x$ and $S x_{2 n} \rightarrow S x$. Since $X$ is Hausdorff, $A x=z=S x$. Because $[A, S]$ is weakly compatible, $A S x=S A x$ and so $A z=S z$. From $(i i), A S x_{2 n} \rightarrow A S x$ and hence $A S x_{2 n} \rightarrow S z$. Also, from continuity of $S$, $S S x_{2 n} \rightarrow S z$.

From (iv),

$$
\begin{aligned}
& M\left(A S x_{2 n}, B x_{2 n-1}, q t\right) \\
& \geq M\left(S S x_{2 n} * T x_{2 n-1}, t\right) * M\left(A S x_{2 n}, S S x_{2 n}, t\right) \\
& * M\left(B x_{2 n-1}, T x_{2 n-1}, t\right) * M\left(A S x_{2 n}, T x_{2 n-1}, t\right) .
\end{aligned}
$$

Taking limit as $n \rightarrow \infty$,

$$
\begin{aligned}
& M(S z, z, q t) \\
& \geq M(S z, z, t) * M(S z, S z, t) * M(z, z, t) * M(S z, z, t) \\
& \geq M(S z, z, t) .
\end{aligned}
$$

From lemma 2.8, we get $S z=z$, and hence

$$
A z=S z=z .
$$

Since $A X \subset T X$, there exists $v \in X$ such that $T v=A z=z$.

From $(i v)$,

$$
\begin{aligned}
& M\left(A x_{2 n}, B v, q t\right) \\
& \geq M\left(S x_{2 n}, T v, t\right) * M\left(A x_{2 n}, S x_{2 n}, t\right) \\
& * M(B v, T v, t) * M\left(A x_{2 n}, T v, t\right) .
\end{aligned}
$$


Letting $n \rightarrow \infty$, we have

$$
\begin{aligned}
& M(z, B v, q t) \\
& \geq M(z, T v, t) * M(z, z, t) \\
& * M(B v, T v, t) * M(z, T v, t) \\
& =M(z, z, t) * M(z, z, t) \\
& * M(B v, z, t) * M(z, z, t) \\
& \geq M(B v, z, t)
\end{aligned}
$$

and so $B v=z$ and hence $T v=B v=z$. Since $[B, T]$ is weakly compatible, $T B v=B T v$ and hence

$$
T z=B z
$$

From $(i v)$,

$$
\begin{aligned}
& M\left(A x_{2 n}, B z, q t\right) \\
& \geq M\left(S x_{2 n}, T z, t\right) * M\left(A x_{2 n}, S x_{2 n}, t\right) \\
& * M(B z, T z, t) * M\left(A x_{2 n}, T z, t\right)
\end{aligned}
$$

which implies that taking limit as $n \rightarrow \infty$,

$$
\begin{aligned}
& M(z, B z, q t) \\
& \geq M(z, T z, t) * M(z, z, t) \\
& * M(B z, T z, t) * M(z, T z, t) \\
& =M(z, B z, t) * M(z, z, t) \\
& * M(B z, B z, t) * M(z, B z, t) \\
& \geq M(z, B z, t)
\end{aligned}
$$

which imlies that

$$
B z=z .
$$

From (3.1.1), (3.1.2) and (3.1.3), A, B, S and $T$ have common fixed point $z$ in $X$. Then

For uniqueness, let $w$ be another common fixed point of $A, B, S$ and $T$.

$$
\begin{aligned}
& M(z, w, q t)=M(A z, B w, q t) \\
& \geq M(S z, T w, t) * M(A z, S z, t) * M(B w, T w, t) * M(A z, T w, t) \\
& \geq M(z, w, t) .
\end{aligned}
$$

From lemma 2.8, $z=w$. 
Corollary 3.2. Let $(X, M, *)$ be a complete $\epsilon$-chanable fuzzy metric space and let $A, B, S$ and $T$ be self mappings of $X$ satisfying $(i)-($ iii $)$ of theorem 3.1 and there exists $q \in(0,1)$ such that $M(A x, B y, q t) \geq M(S x, T y, t) *$ $M(A x, S x, t) * M(S x, B y, 2 t) * M(B y, T y, t) * M(T y, A x, t)$, for every $x, y \in X$ and $t>0$.

Then $A, B, S$ and $T$ have a unique common fixed point in $X$.

Proof. From definition, we have that $M(S x, T y, t) * M(A x, S x, t) * M(B y, T y, t) *$ $M(B y, S x, 2 t) * M(A x, T y, t) \geq M(S x, T y, t) * M(A x, S x, t) * M(B y, T y, t) *$ $M(S x, T y, t) * M(T y, B y, t) * M(A x, T y, t) \geq M(S x, T y, t) * M(A x, S x, t) *$ $M(B y, T y, t) * M(A x, T y, t)$ and hence, from theorem 3.1, A, B, S and $T$ have a unique fixed point in $X$.

Corollary 3.3. Let $(X, M, *)$ be a complete $\epsilon$-chanable fuzzy metric space and let $A, B, S$ and $T$ be self mappings of $X$ satisfying $(i)-($ iii) of theorem 3.1 and there exists $q \in(0,1)$ such that $M(A x, B y, q t) \geq M(S x, T y, t)$, for every $x, y \in X$ and $t>0$.

Then $A, B, S$ and $T$ have a unique common fixed point in $X$.

Proof. e have that $M(S x, T y, t)=M(S x, T y, t) * 1=M(S x, T y, t) * M(A x, A x, 5 t)$

$\geq M(S x, T y, t) * M(A x, S x, t) * M(S x, B y, 2 t) * M(B y, T y, t) * M(T y, A x, t)$ and hence, from corollary 3.2, $A, B, S$ and $T$ have a unique fixed point in $X$.

Let $S$ and $T$ be the identity mapping on $X$ in corollary 3.3. Then we get the next result.

Corollary 3.4. Let $(X, M, *)$ be a complete $\epsilon$-chanable fuzzy metric space and let $A$ and $B$ be self mappings of $X$ satisfying the following condition: there exists $q \in(0,1)$ such that $M(A x, B y, q t) \geq M(x, y, t)$, for every $x, y \in X$ and $t>0$.

Then $A$ and $B$ have a unique common fixed point in $X$.

In corollary 3.4, if we take $A=B$, then this result becomes to fuzzy Banach contraction theorem.

Corollary 3.5. Let $(X, M, *)$ be a complete $\epsilon$-chanable fuzzy metric space and let $A$ be self mapping of $X$ satisfying the following condition:

there exists $q \in(0,1)$ such that $M(A x, A y, q t) \geq M(x, y, t)$ for every $x, y \in$ $X$ and $t>0$.

Then $A$ has a unique fixed point in $X$.

Let $(X, M, *)$ be a complete $\epsilon$-chanable fuzzy metric space. For any $x, y \in$ $X$ and $t>0$, let $M_{\epsilon}(x, y, t)=\sup _{c}\left\{M\left(x_{0}, x_{1}, t\right) * M\left(x_{1}, x_{2}, t\right) * \cdots * M\left(x_{n-1}, x_{n}, t\right) \mid c:\right.$ $x=x_{0}, x_{1}, \cdots, x_{n}=y$ is an $\epsilon$-chain from $x$ to $\left.y\right\}$. Then it is easy to show that $M_{\epsilon}$ is fuzzy metric satisfying $M(x, y, t) \geq M_{\epsilon}(x, y, t)$ and $M(x, y, t)=$ $M_{\epsilon}(x, y, t)$, whenever $M(x, y, t)>1-\epsilon$. 
Lemma 3.6. Let $(X, M, *)$ be a complete $\epsilon$-chanable fuzzy metric space. Then $\left(X, M_{\epsilon}, *\right)$ is complete $\epsilon$-chanable.

Proof. Let $x, y \in X$. Since $(X, M, *)$ is $\epsilon$-chanable, there exists a finite sequence $x=x_{0}, x_{1}, \cdots, x_{n}=y$ such that $M\left(x_{i-1}, x_{i}, t\right)>1-\epsilon$, and hence $M_{\epsilon}\left(x_{i-1}, x_{i}, t\right)=M\left(x_{i-1}, x_{i}, t\right)>1-\epsilon$. Thus $\left(X, M_{\epsilon}, *\right)$ is $\epsilon$-chanable.

Now, we show that $\left(X, M_{\epsilon}, *\right)$ is complete.

Let $\left\{x_{n}\right\}$ be a Cauchy sequence in $\left(X, M_{\epsilon}, *\right)$. Then $M_{\epsilon}\left(x_{m}, x_{n}, t\right)>1-\epsilon$ for $m>n$. Since $(X, M, *)$ is $\epsilon$-chanable, there exists $\epsilon$-chain $x_{n}=y_{1}, y_{2}, \cdots, y_{l}=$ $x_{m}$ such that $M\left(y_{i-1}, y_{i}, y\right)>1-\epsilon$ for $i=1,2, \cdots, l$. Hence $M\left(x_{n}, x_{m}, t\right) \geq$ $M\left(y_{1}, y_{2}, t / l\right) * \cdots * M\left(y_{l-1}, y_{l}, t / l\right)>(1-\epsilon) * \cdots *(1-\epsilon) \geq 1-\epsilon$, and hence $M_{\epsilon}\left(x_{n}, x_{m}, t\right)=M\left(x_{n}, x_{m}, t\right)$. Since $(X, M, *)$ is complete, there exists $z \in X$ such that $M\left(x_{n}, z, t\right)>1-\epsilon$ and hence $M_{\epsilon}\left(x_{n}, z, t\right)=M\left(x_{n}, z, t\right)$ and $M_{\epsilon}\left(x_{n}, z, t\right)>1-\epsilon$. Hence $\left\{x_{n}\right\}$ converges to $z$ and $\left(X, M_{\epsilon}, *\right)$ is complete.

Theorem 3.7. Let $(X, M, *)$ be a complete $\epsilon$-chanable fuzzy metric space and let $T_{1}$ and $T_{2}$ be self mappings of $X$ satisfying the following condition:

If $M(x, y, t)>1-\epsilon$, there exists $q \in(0,1)$ such that for $i, j=1,2$, $M\left(T_{i} x, T_{j} y, q t\right) \geq M(x, y, t)$ for all $x, y \in X$ and $t>0$. Then $T_{1}$ and $T_{2}$ have a unique common fixed point in $X$.

Proof. For any $x, y \in X$ and $t>0$, let $M_{\epsilon}(x, y, t)=\sup _{c}\left\{M\left(x_{0}, x_{1}, t\right) *\right.$ $M\left(x_{1}, x_{2}, t\right) * \cdots * M\left(x_{n-1}, x_{n}, t\right) \mid c: x=x_{0}, x_{1}, \cdots, x_{n}=y$ is an $\epsilon$-chain from $x$ to $y\}$. Then we know that $M(x, y, t)=M_{\epsilon}(x, y, t)$, whenever $M(x, y, t)>1-\epsilon$.

Given $x, y \in X$ and $\epsilon$-chain $c: x=x_{0}, x_{1}, \cdots, x_{n}=y$ such that $M\left(x_{i}, x_{i-1}, t\right)>$ $1-\epsilon$, we have that $M\left(T_{1} x_{0}, T_{1} x_{1}, q t\right) \geq M\left(x_{0}, x_{1}, t\right)>1-\epsilon$ and $M\left(T_{2} x_{2}, T_{1} x_{1}, q t\right) \geq$ $M\left(x_{2}, x_{1}, t\right)>1-\epsilon$, and hence $T_{1} x_{0}, T_{1} x_{1}, T_{2} x_{2}$ form an $\epsilon$-chain from $T_{1} x_{0}$ to $\mathrm{T}_{2} x_{2}$.

Similarly, if $n$ is odd(say $\mathrm{n}=3$ ), we can show that $T_{1} x_{0}, T_{2} x_{1}, T_{1} x_{2}$ and $T_{2} x_{3}$ form an $\epsilon$-chain from $T_{1} x_{0}$ to $T_{2} x_{3}$. Thus we have $M_{\epsilon}\left(T_{1} x_{0}, T_{2} x_{3}, q t\right) \geq$ $M\left(T_{1} x_{0}, T_{2} x_{1}, q t\right) * M\left(T_{2} x_{1}, T_{1} x_{2}, q t\right) * M\left(T_{1} x_{2}, T_{2} x_{3}, q t\right) \geq M\left(x_{0}, x_{1}, t\right) * M\left(x_{1}, x_{2}, t\right) *$ $M\left(x_{2}, x_{3}, t\right)$. Hence it follows that $M_{\epsilon}\left(T_{1} x, T_{2} y, q t\right) \geq M\left(x_{0}, x_{1}, t\right) * M\left(x_{1}, x_{2}, t\right) *$ $\cdots, * M\left(x_{n-1}, x_{n}, t\right)$, where $x=x_{0}, x_{1}, \cdots, x_{n}=y$ is an arbitrary $\epsilon$-chain from $x$ to $y$. Thus we have $M_{\epsilon}\left(T_{1} x, T_{2} y, q t\right) \geq M_{\epsilon}(x, y, t)$. From lemma 3.6 and corollary $3.4, T_{1}$ and $T_{2}$ have a unique common fixed point in $X$.

In the following, we extend the result of [5] in metric space to $\epsilon$-chanable fuzzy metric space.

Theorem 3.8. Let $(X, M, *)$ be a complete $\epsilon$-chanable fuzzy metric space and let $S$ be continuous self mapping of $X$ and $T$ be self mapping of $X$. Then $S$ and $T$ have a common fixed point in $X$ if and only if there exists a contnuous self mapping $A$ of $X$ such that the following conditions are satisfied:

(i) $A X \subset T X \cap S X$, 
(ii) the pairs $[A, S]$ and $[A, T]$ are weakly compatible,

(iii) there exists $q \in(0,1)$ such that for every $x, y \in X$ and $t>0$, $M(A x, A y, q t) \geq M(S x, T y, t) * M(A x, S x, t) * M(A y, T y, t) * M(A x, T y, t)$. In fact $A, S$ and $T$ have a unique common fixed point in $X$.

Proof. First, we show that the necessity of the conditions $(i)-(i i i)$. Suppose that $S$ and $T$ have a common fixed point in $X$, say $z$. Then $S z=z=T z$.

Let $A x=z$ for all $x \in X$. Then $A X \subset T X \cap S X$. Since $A S z=S A z$ and $A T z=T A z,[A, S]$ and $[A, T]$ are weakly compatible and hence the conditions (i) and $($ ii $)$ are satisfied.

For some $q \in(0,1)$, we have that

$M(A x, A y, q t)=1 \geq M(S x, T y, t) * M(A x, S x, t) * M(A y, T y, t) * M(A x, T y, t)$ for every $x, y \in X$ and $t>0$, and hence the condition (iii) is satisfied.

Now, for the sufficiency of the conditions, let $A=B$ in theorem 3.1. Then $A, S$ and $T$ have a unique common fixed point in $X$.

Corollary 3.9. Let $(X, M, *)$ be a complete $\epsilon$-chanable fuzzy metric space and let $S$ be continuous self mapping of $X$ and $T$ be self mapping of $X$. Then $S$ and $T$ have a common fixed point in $X$ if and only if there exists a continuous self mapping $A$ of $X$ satisfying (i)-(ii) of theorem 3.8 and there exists $q \in(0,1)$ such that for every $x, y \in X$ and $t>0$,

$M(A x, A y, q t) \geq M(S x, T y, t) * M(A x, S x, t) * M(A y, T y, t) * M(A y, S x, 2 t) *$ $M(A x, T y, t)$.

In fact $A, S$ and $T$ have a unique common fixed point in $X$.

Corollary 3.10. Let $(X, M, *)$ be a complete $\epsilon$-chanable fuzzy metric space and let $S$ be continuous self mapping of $X$ and $T$ be self mapping of $X$. Then $S$ and $T$ have a common fixed point in $X$ if and only if there exists a continuous self mapping $A$ of $X$ satisfying $(i)-(i i)$ of theorem 3.8 and there exists $q \in$ $(0,1)$ such that for every $x, y \in X$ and $t>0, M(A x, A y, q t) \geq M(S x, T y, t)$.

In fact $A, S$ and $T$ have a unique common fixed point in $X$.

\section{References}

[1] S. H. Cho and S. C. Kim, On common fixed points in fuzzy metric spaces through weak compatibility, Internat. J. Pure and Appl. Math., to appear.

[2] Deng Zi-ke, Fuzzy pseudo metric spaces, J. Math. Anal. Appl., 86 (1982), 74-95.

[3] B. C. Dhage, On common fixed point of coincidentally commuting mappings in D-metric spaces, Indian J. Pure Appl. Math., 30 (1999), 395-406. 
[4] A. Erceg, Metric spaces in fuzzy set theory, J. Math. Anal. Appl., 69(1979), 205-230.

[5] B. Fisher, Mappings with a common fixed point, Math. Seminar Notes Kobe Univ., 7 (1979), 81-84.

[6] A. George and P. Veeramani, On some results in fuzzy metirc spaces, Fuzzy sets and Systems, 64 (1994), 395-399.

[7] A. George and P. Veeramani, On some results of analysis for fuzzy metirc spaces, Fuzzy sets and Systems, 90 (1997), 365-368.

[8] M. Grabiec, Fixed points in fuzzy metric spaces, Fuzzy sets and Systems, 27 (1988), 245-252.

[9] Valentin Gregori and Almanzor Sapena, On fixed point theorems in fuzzy metric spaces, Fuzzy sets and Systems, 125(2002), 245-252.

[10] G. Jungck, Compatible mappings and fixed points, Internat. J. Math. Math. Sci., 9(4)(1986), 771-779.

[11] G. Jungck and B. E. Rhoades, Fixed points for set valued functions without continuity, Indian J. Pure Appl. Math., 29 (1998), 227-238.

[12] O. Kaleva and S. Seikkala, On fuzzy metric spaces, Fuzzy sets and Systems, 12 (1984), 215-229.

[13] E. P. Klement, R. Mesiar and E. Pap, Triangular Norm, Kluwer Academic Publishers, Tren 8.

[14] O. Kramosil and J. Michalek, Fuzzy metric and statistical metric spaces, Kybernetica, 11 (1975), 326-334.

[15] R. N. Mukherjee, On some fixed point theorems, Kyungpook Math. J., 14 (1974), 37-44.

[16] B. Schweizer and A. Sklar, Statistical metric spaces, Pacific J. Math., 10 (1960), 314-334.

[17] S. Sessa, On weak commutativity condition of mappings in fixed point considerations, Publ. Inst. Math. Beograd, 32(46) (1982), 149-153.

[18] Bijendra Singh and M. S. Chauhan, Common fixed points of compatible maps in fuzzy metric spases, Fuzzy sets and Systems, 115 (2000), 471475 .

[19] Bijendra Singh and Shishir Jain, A fixed point theorem in Menger space through weak compatibility, J. Math. Anal. Appl., 301 (2005), 439-448. 
[20] Guangxing Song, Comment on " A common fixed point theorem in a fuzzy metric space", Fuzzy Set and Systems, 135 (2003), 409-413.

[21] L. A. Zadeh, Fuzzy sets, Inform. and Control, 8 (1965), 338-353.

Received: October 27, 2005 